\title{
Efeitos das combinações da prática constante e variada na aquisição de uma habilidade motora
}

Rejane PAROLI ${ }^{*}$ Go TANI*
*Escola de Educação Física e Esporte, Universidade de São Paulo.

\section{Resumo}

Os efeitos de diferentes estruturas de prática na aquisição de uma habilidade motora foram investigados em um experimento em que a prática foi manipulada no que se refere à variabilidade. 0 delineamento constou de dois grupos de prática combinada (constante-por blocos e constante-aleatória) e de duas fases de aprendizagem (estabilização e adaptação). Sessenta estudantes universitários praticaram uma tarefa que consistia em tocar sensores de forma sequencial em integração a um estímulo visual. Para a realização da tarefa foi utilizado o aparelho de "timing" coincidente para tarefas complexas. 0 desempenho foi avaliado mediante três medidas de erro: absoluto, variável e de execução. 0 "timing" relativo e sua variabilidade foram utilizados para avaliar a macroestrutura do programa de ação da habilidade ao passo que a microestrutura foi avaliada pela variabilidade do tempo total de movimento. Os resultados mostraram que, ao variar a tarefa em seus aspectos perceptivo (velocidade do estímulo visual) e efetor (sequência de toques), a prática constante seguida de prática por blocos apresenta, na fase de adaptação, melhor desempenho em relação ao número de acertos de execução do que a prática constante seguida de prática aleatória, indicando, portanto, que a primeira proporciona mais condições de adaptação a uma nova tarefa motora.

Unitermos: Estrutura de prática; Aquisição de habilidades motoras; Processo adaptativo.

\section{Introdução}

A prática e o "feedback" são fatores determinantes na aprendizagem de novas habilidades motoras e por isso merecem atenção especial da área de estudo de Aprendizagem Motora.

Com relação à prática, especificamente com relação à estruturação da prática, vários estudos foram realizados motivados pelas implicaçōes da teoria de esquema e da abordagem da interferência contextual e têm demonstrado que o suporte empírico das prediçôes não é tão consistente quanto acreditava-se. Revisões de literatura mostraram que a superioridade da prática variada sobre a constante foi confirmada em menos da metade dos estudos analisados quando testada a predição da hipótese da variabilidade de prática (ver VAN Rossum, 1990) e o mesmo ocorreu com os estudos testando a superioridade da prática aleatória sobre a prática por blocos (ver MeIRA JúNIOR, 1999).

Para melhor entender esses resultados, uma mudança significativa nos estudos atuais sobre estrutura de prática foi à adoção de medidas referentes à estrutura interna que se supóe controlar os movimentos (CORRÊA, 2001; CORRÊA, BENDA, MeIRA JúNIOR \& TANI, 2003; CORRÊA, BENDA \& TANI, 2001; Corrêa, Barros \& Massigli \& Gonçalves, 2007; Corrêa, Gonçalves, Barros \& Massigli, 2006; Lai \& ShEA, 1998; LAI, SHEA, BRUeCHERT \& LitTLe, 2002; Lai, SheA, Wulf \& WRight, 2000; ShEA, LaI, Wright, IMMINK \& BLACK, 2001; UGRINOWITSCH, 2003; WuLF, 1992; Wulf \& LeE, 1993).

Adotando essas medidas, Wulf (1992), Wulf e LEE (1993) e LAI et al. (2002) investigaram o efeito da interferência contextual e encontraram padrōes de dissociação contraditórios ao utilizar uma tarefa de "timing". Wuf e LeE (1993) mostraram que a prática variada seriada era mais efetiva do que a por blocos no desenvolvimento do programa motor generalizado (PMG), por outro lado, não havia diferenças significantes entre os grupos em relação às 
medidas de parâmetro. Com relação ao desempenho, o grupo de prática por blocos apresentava uma superioridade de significância marginal. $\mathrm{O}$ estudo de WULF (1992) mostrou que as duas práticas eram equivalentes no desenvolvimento do PMG, porém a prática aleatória proporcionava uma melhor parametrização do movimento e um melhor desempenho do que a prática por blocos. Já o estudo de LAI et al. (2002) mostrou que a prática por blocos proporcionava melhores resultados que a aleatória para o desenvolvimento do programa e benefícios semelhantes para a parametrização de movimento.

Diante desses resultados inconclusivos, SHEA et al. (2001) retomaram a questão da variabilidade de prática, utilizando quatro grupos experimentais: constante, por blocos, seriada e aleatória. Apesar da data de publicação ser anterior ao do estudo de LAI et al. (2002), esse estudo foi realizado posteriormente. Os resultados mostraram que o grupo constante e por blocos produziram menos erros do que o grupo de prática aleatória para a medida de "timing" relativo, ao passo que o grupo de prática seriada apresentou pior desempenho em relação ao grupo de prática constante. Para as medidas de parametrização, os erros eram consideravelmente maiores para os grupos de prática constante e por blocos do que para os grupos de prática seriada e aleatória.

Portanto, o estudo de SHEA et al. (2001) replicou os resultados de LAI et al. (2002), o que indica uma tendência das práticas que oferecem mais repetições do mesmo movimento favorecerem o desenvolvimento do PMG e das práticas que oferecem mais variabilidade da resposta favorecerem a parametrização do movimento.

Um outro grupo de pesquisadores, também adotando medidas referentes à estrutura formada com a prática, chegou à conclusão semelhante ao realizar pesquisas dentro de uma outra perspectiva teórica denominada processo adaptativo.

$\mathrm{Na}$ abordagem de Processo Adaptativo consideramse duas fases: a estabilização e a adaptação. Segundo TANI $(1995,2005)$, a estabilização representa um processo de busca por uma padronização espaçotemporal dos movimentos. Assim, a inconsistência e a falta de coordenação iniciais são gradualmente eliminadas e substituídas por movimentos padronizados e precisos, a partir dos quais se infere a formação da estrutura. A outra fase considera a adaptação a novas situações ou a novas tarefas motoras através da aplicação das habilidades motoras já adquiridas. As adaptações podem ocorrer por meio de modificações na estrutura da habilidade estabelecida que resultem numa mudança qualitativa no sistema em um nível superior de complexidade ou podem ser alcançadas por meio de sua flexibilidade inerente, fazendo uso da redundância do sistema (TANI, 1995, 2005).

A estabilização ocorre num ciclo sucessivo sempre em direção a um estado de organização mais complexo do que o anterior. Para desencadear esses ciclos sucessivos de estabilidade são necessários períodos de instabilidade em que a perturbação exerce função fundamental no processo de mudanças. A estabilização ocorre num ciclo sucessivo. Entretanto, para possibilitar adaptações às demandas ambientais é necessário que a estrutura formada também possua características flexíveis (Choshi, 1986 citado por TANI, 1995, 2005). Com base nessa ideia, a estrutura da habilidade foi concebida como uma estrutura hierárquica constituída de dois níveis complementares: o da macroestrutura e o da microestrutura. Enquanto a macroestrutura está orientada à ordem e é responsável pela consistência do desempenho, a microestrutura orienta-se à desordem e fica responsável pela sua flexibilidade (MANOEL, 1992; Manoel, Basso, Corrêa \& Tani, 2002; Manoel \& Connolly, 1995; TANI, 1995, 2005)

Assim sendo, a interação entre os componentes de uma ação em um comportamento motor estabilizado é invariante, bem definida e implica num padrão geral da ação (macroestrutura). Aspectos como "timing" relativo, força relativa e sequência de componentes respondem pelos aspectos invariáveis de um programa de ação.

Já quanto à microestrutura, o comportamento não é definido e não segue um padrão geral, implicando na possibilidade de assumir diferentes interaçōes, cada uma dirigida a alcançar diferentes efeitos no ambiente. O tempo total de movimento e a força total são alguns dos aspectos do programa considerados como variáveis, portanto aqueles que provêem uma configuração específica para cada resposta (MANOEL \& CONNOLlY, 1995; TANI, 1995). Tanto os aspectos invariáveis quanto os variáveis dos programas de ação podem sofrer alteraçōes de acordo com as características da tarefa, o nível de proficiência do indivíduo e do estágio de desenvolvimento (TANI, 1995).

Alguns estudos já foram desenvolvidos dentro da perspectiva do processo adaptativo investigando a questão da estrutura de prática (CORRÊA, 2001; CORRÊA etal., 2003, 2006, 2007; CoRrêA, BENDA \& TANI, 2001).

Dentre eles destaca-se o estudo de CORRÊA (2001) que investigou os efeitos da prática constante e aleatória e de suas combinações na aquisição de uma habilidade motora, manipulando os aspectos temporal 
(experimento 1), espacial (experimento 2) ou ambos os aspectos da tarefa (experimento 3). Segundo o autor, os resultados mostraram que a prática constante, aleatória e suas combinações tiveram efeitos semelhantes nas medidas de desempenho. No entanto, os resultados permitiram a inferência de que a prática constante seguida pela prática aleatória possibilitou a formação de uma estrutura mais flexível do que os demais tipos de prática. Isto porque as crianças do grupo de prática constante-aleatória adaptaram-se sem modificação da macroestrutura da habilidade, recurso a que as crianças dos demais grupos tiveram que recorrer. Portanto, no seu conjunto, os resultados apontam para um melhor efeito da prática constante seguida da prática aleatória no processo adaptativo.

É de realçar que, apesar de partir de abordagens teóricas diferentes, estudos recentes sobre estrutura de prática (CORRÊA, 2001; LAI et al., 2000) indicam que a prática constante ou mais repetitiva seguida de uma prática variada otimizaria o processo de aquisição de habilidades motoras, o que pode ser um indício de consistência dos resultados. Porém, uma limitação dos estudos realizados dentro da perspectiva do PMG é a utilização de uma visão finita do processo de aprendizagem motora. Isso pode ser verificado na interpretação dos resultados de que a melhor prática é aquela que propicia a manutenção ou o alcance de uma estrutura préestabelecida. Tais estudos não buscam saber como o indivíduo adapta-se às novas situações, quais estratégias são utilizadas ou quais adaptações são feitas frente a diferentes condições ou, em outras palavras, como as novas estruturas são formadas a partir daquelas já existentes (estabilizadas). Devido a isso, que nesse estudo adota-se a visão do Processo
Adaptativo que encara a aprendizagem motora como um processo de sucessivos ciclos de instabilidade - estabilidade - instabilidade.

Cabe destacar que os estudos citados utilizaram a prática aleatória como prática variada. Todavia existem outras possibilidades de variar a prática como com uma estruturação por blocos, por exemplo. Portanto, surge a dúvida: será que a prática por blocos oferecida após a prática constante proporcionaria os mesmos resultados? Será que a prática que oferece mais instabilidade é a mais adequada?

$\mathrm{O}$ modelo adotado propóe que elementos como a incerteza, a aleatoriedade e a instabilidade são importantes no processo de aprendizagem (BARROCAL, Perez, Meira Junior, Gomes \& Tani, 2006; Bastos, Araújo, Walter \& Freudenheim, 2007; Benda, 2001; Benda, Corrêa, Oliveira \& Tani, 2000; Benda, Corrêa, Oliveira, Ugrinowitsch \& Tani, 2005; CorrêA, 2001; CorrêA et al., 2006, 2007; Manoel \& Connolly, 1995; Tani, 1995, 2000; Tani, Meira Junior \& Gomes, 2005; UGrinOWISTCH, 2003; Ugrinowitsch, Coca, Tertuliano, Gimenez \& Pereira, 2003), principalmente se inseridas após a formação inicial de uma estrutura, porém não se sabe o quanto de instabilidade, de incerteza e aleatoriedade é preciso.

Uma das formas de controlar essa quantidade de incerteza pode ser através da utilização da prática por blocos ou aleatória, a primeira proporcionando menos incerteza que a segunda.

Portanto, o objetivo desse estudo foi investigar o efeito da combinação da prática variada, aleatória ou por blocos, na aquisição de uma habilidade de timing coincidente quando combinadas com a prática constante.

\section{Método}

\section{Participantes}

Participaram desse experimento 60 universitários voluntários, de ambos os sexos, com média de idade de aproximadamente 24 anos e desvio-padrão de cinco anos, sem experiência prévia na tarefa. Eles foram distribuídos em dois grupos experimentais, cada um dos grupos composto de 16 homens e 14 mulheres.

\section{Tarefa e aparato}

O instrumento utilizado foi o aparelho de "timing" coincidente em tarefas complexas utilizado em outros estudos (CORRÊA et al., 2006, 2007; FrEUdENHEIM, Oliveira, Corrêa, Oliveira, Dantas, Moreira \& TANi, 2005; Pinheiro \& CorrêA, 2005; UGRINOWISTCH, CORRÊA \& TANI, 2005) e patenteado sobre o número P.I. 0.403.433-4. Esse aparelho é 
composto por uma canaleta de 195 centímetros de comprimento, oito centímetros de largura e dois centímetros de altura. Sobre a canaleta estão dispostos 96 diodos com distância de um centímetro e meio entre eles. Essa canaleta é semelhante à canaleta de Bassin Antecipation Timer e foi posicionada num ângulo de 30 graus (PAYNE, 1987).

Também compõem o equipamento, uma mesa de madeira (71 centímetros de comprimento, 43,5 centímetros de largura e seis centímetros de altura) sobre a qual estão dispostos quatro sensores (medindo cinco centímetros de largura e 15 centímetros de comprimento) e o computador com um "software" que possibilita o acendimento dos diodos em sequência, em diferentes velocidades e com mudança de aceleração. O equipamento também conta com um indicador visual de conhecimento de resultados que qualifica a performance por meio de cinco luzes: uma verde (correto), duas amarelas (a da esquerda - um pouco antes e a da direita- um pouco depois) e duas vermelhas (esquerda - muito antes, direita - muito depois). A luz verde indicando correto acendia quando o participante tocasse o sensor alvo até 30 ms antes ou depois da chegada do estímulo visual. A luz amarela indicando um pouco antes acendia quando o participante tocasse o sensor alvo entre $30 \mathrm{~ms}$ e $60 \mathrm{~ms}$ antes da chegada do estímulo visual e a luz amarela indicando um pouco depois quando esse toque era realizado entre $30 \mathrm{~ms}$ e $60 \mathrm{~ms}$ depois da chegada do estímulo visual. Da mesma forma, uma das luzes vermelhas acendia quando o movimento era realizado entre 60 e 90 ms antes da chegada do estímulo (muito antes) ou depois da sua chegada (muito depois). Os tempos entre os toques em cada sensor são registrados.

Assim, a tarefa consistiu em realizar uma sequência de cinco toques (SQ1 = 1-2-3-1-4), de modo que o último toque coincidisse com o acendimento de um diodo alvo (FIGURA 1)

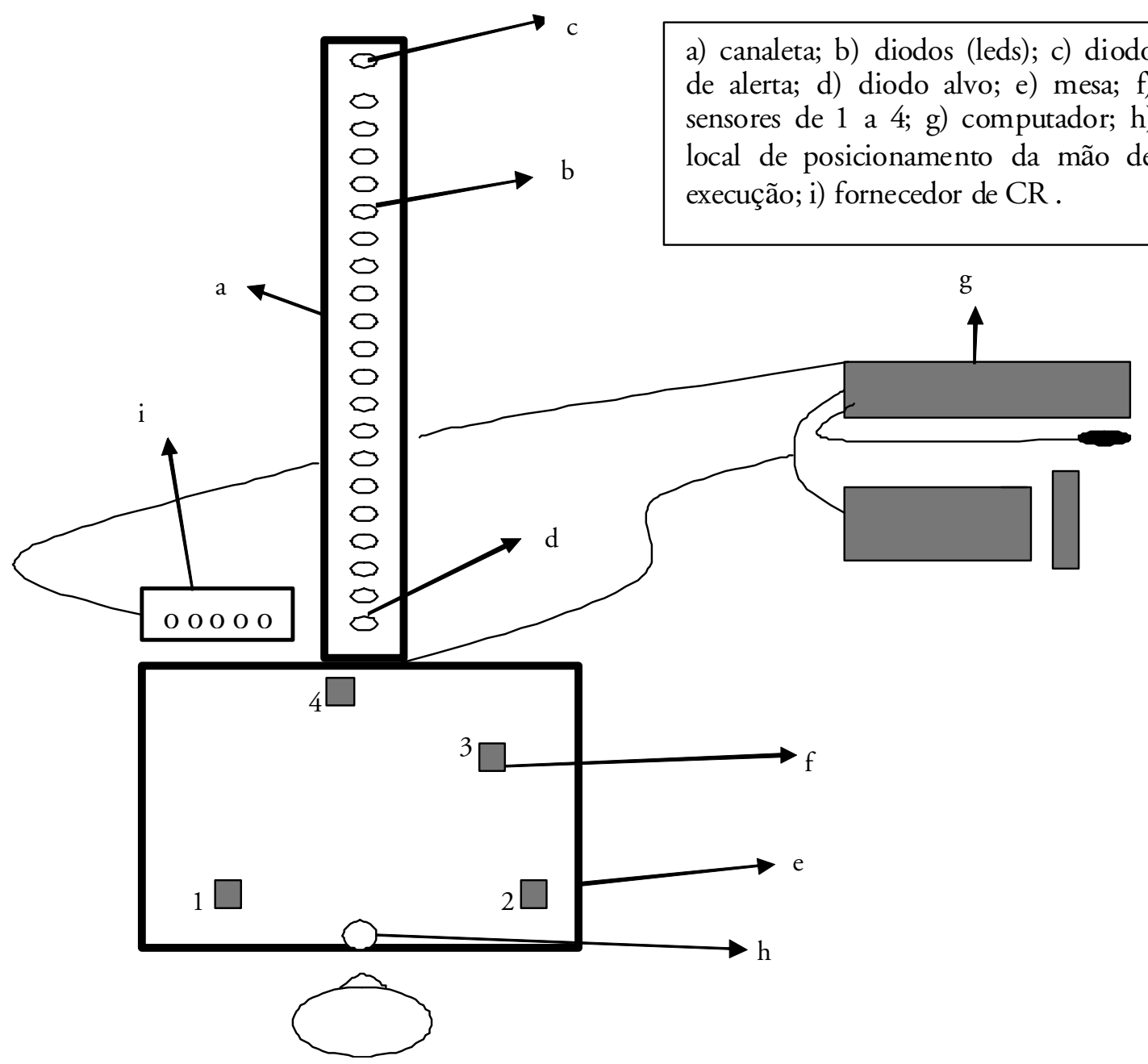

FIGURA 1 - Ilustração esquemática do aparelho de "timing" coincidente em tarefas complexas. 
A partir desses dados foram calculadas e utilizadas as seguintes medidas de desempenho: erro absoluto (mediana da diferença absoluta entre o desempenho alcançado e a meta), erro variável (coeficientes de variação de Pearson intra-sujeitos das diferenças absolutas entre o desempenho alcançado e a meta), número de acertos de execução (mediana do número de tentativas executadas na sequência estabelecida).

Foram utilizadas, também, medidas referentes ao padrão de execução como: "timing" relativo e o tempo total de movimento que representam os aspectos da macroestrutura e da microestrutura da tarefa respectivamente. $\mathrm{O}$ "timing relativo" (TR) foi calculado em relação à magnitude (mediana) e variabilidade (coeficiente de variação de Pearson) dos componentes, sendo que a primeira mostra a proporção dos componentes em relação ao tempo total e a segunda indica a variabilidade dessa proporção. Com relação à medida de tempo total utilizou-se o coeficiente de variação do tempo total de movimento como medida representante da variabilidade da microestrutura da habilidade. Não são apresentadas medidas relativas à magnitude da microestrutura, pois a medida de variabilidade já é suficiente para demonstrar a variação entre tentativas, que tem maior relevância para a análise.

\section{Delineamento e procedimentos}

O estudo envolveu os grupos de prática constante seguida de prática aleatória (PCA) e de prática constante seguida de prática por blocos (PCB). O experimento foi realizado em duas fases experimentais: a fase de estabilização e a fase de adaptação. $\mathrm{Na}$ primeira, os grupos praticaram a tarefa de forma constante até atingir o valor critério de três tentativas consecutivas com erro de até $30 \mathrm{~ms}$. Esse procedimento foi adotado para assegurar que os sujeitos começassem a próxima etapa na mesma fase de aprendizagem (independentemente do número de tentativas necessárias para alcançá-la), além de servir como controle de possíveis experiências anteriores com tarefas similares. Depois disso, realizaram mais 36 tentativas de forma variada aleatória ou por blocos, de acordo com a situação experimental de cada grupo, com mudança na sequência de toques e na velocidade do estímulo visual (mudança perceptiva-motora). Foi fornecido conhecimento de resultado (CR) em todas as tentativas da fase de estabilização.

$\mathrm{Na}$ segunda fase, foram executadas mais 27 tentativas com uma velocidade, sequência e fornecimento de CR diferente das utilizadas na fase anterior.

Os dois grupos executaram as tentativas de prática constante com uma única velocidade de estímulo visual $(\mathrm{V} 1=142,86 \mathrm{~cm} / \mathrm{s})$ numa única sequência de toques $(\mathrm{SQ1}=1-2-3-1-4)$. Já durante a prática variada executaram 36 tentativas combinando três diferentes velocidades $(\mathrm{V} 1=142,86 \mathrm{~cm} / \mathrm{s}, \mathrm{V} 2=166,67 \mathrm{~cm} / \mathrm{s}$, $\mathrm{V} 3=125 \mathrm{~cm} / \mathrm{s}$ ) e sequências (SQ1 = 1-2-3-1-4, SQ2 = 1-3-2-3-4 e SQ3 = 1-3-2-1-4). Na fase de adaptação, os dois grupos executaram as tentativas em uma nova sequência de toques (SQ4 = 1-3-1-2-4) e numa nova velocidade $(\mathrm{V} 4=111,11 \mathrm{~cm} / \mathrm{s})$. As velocidades do estímulo visual eas sequências de toques adotadas foram semelhantes às utilizadas nos estudos anteriores (CORRÊA, 2001; CORRÊA et al., 2006, 2007) e se mostraram adequadas em termos de nível de dificuldade e possibilidade de realização. Antes do início da coleta, todos os sujeitos assinaram o formulário de consentimento livre e esclarecido aprovado pelo Comitê de Ética da Escola de Educação Física e Esporte da Universidade de São Paulo.

\section{Resultados}

Os dados foram analisados em sete blocos de nove tentativas, os quatro primeiros blocos referentes à prática variada da fase de estabilização e os três últimos blocos referentes à fase de adaptação. Ambos os grupos realizaram a prática constante da mesma forma, até atingir o critério de desempenho, o que os diferenciou foi, justamente, o tipo de prática variada realizada após a constante, por isso, são analisadas apenas as tentativas de prática variada.

Para a realização da análise inferencial, foi garantida a independência entre as observações e testado o pressuposto da igualdade de variância. Se a medida apresentava igualdade de variância em todos os blocos analisados conjuntamente, era realizada a análise paramétrica ANOVA "two-way" com medidas repetidas no último fator, com nível de significância de 0,05 , se a medida não apresentava igualdade de variância em todos os blocos analisados conjuntamente, eram realizados os testes nãoparamétricos de Friedman para cada grupo, a fim de verificar as diferenças entre os blocos de tentativas, e o teste U de Mann Whitney em cada bloco para verificar se havia diferenças entre os grupos. 
Foi realizado o teste "Post Hoc" de Scheffé para localizar as diferenças na análise paramétrica e só foram descritas aquelas com significado teórico, ou seja, diferenças entre os blocos de um mesmo grupo ou entre os grupos no mesmo bloco de tentativas. $\mathrm{Na}$ análise não-paramétrica, comparações emparelhadas foram conduzidas para localizar as diferenças entre os blocos usando o teste de Wilcoxon e controlando o erro tipo 1 através do procedimento sequencial Holm de Bonferroni.

\section{Medidas de desempenho}

As diferenças estatísticas são apresentadas na TABELA 1.

A análise dos resultados mostrou que o $\mathrm{PCB}$ apresentou desempenho mais preciso (menor erro absoluto) e consistente (menor erro variável) que o PCA durante a fase de estabilização. Entretanto, os resultados mostraram que o PCA apresentou melhoras ao longo da prática e conseguiu alcançar o mesmo nível de desempenho do PCB no final dela.
Os dois grupos não mostraram diferenças significativas em relação ao número de acertos na fase de estabilização, foram constatadas apenas diferenças intra-grupo para o PCA que errou mais no primeiro comparado ao segundo e terceiro blocos dessa fase.

$\mathrm{Na}$ fase de adaptação, os dois grupos apresentaram o mesmo comportamento quanto à consistência do desempenho. Ambos apresentaram melhor consistência nos três blocos da adaptação do que no final da estabilização, além de a aumentarem durante a adaptação, do primeiro para o segundo e terceiro blocos. Com relação ao número de acertos, o PCB errou significativamente menos que o PCA no segundo bloco da fase de adaptação.

No que se referem ao erro absoluto, os dois grupos apresentaram o mesmo nível de precisão, porém foram observados comportamentos diferentes para cada grupo ao longo dos blocos. Enquanto o PCB manteve a mesma precisão da fase anterior, o PCA aumentou-a, do final da estabilização para os três blocos da adaptação e do primeiro para último bloco da adaptação (FIGURA 2).

TABELA 1 - Diferenças estatísticas significativas intra-grupo e entre grupos do PCB e do PCA das medidas de erro absoluto, erro variável e número de acertos de execução nos últimos quatro blocos da estabilização (E1 a E4) e entre último bloco da fase de estabilização (E4) e os três blocos da fase de adaptação (A1 a A3).

Na primeira metade da tabela são apresentadas as diferenças estatísticas para cada medida de desempenho relativas à fase de estabilização, na primeira coluna, as diferenças entre os blocos e, na segunda coluna, as diferenças entre os grupos PCB e PCA. Foram conduzidas análises de variância com medidas repetidas no segundo fator (ANOVAs two way) para a medida de erro variável nos quatro blocos da fase de estabilização e entre o último bloco da fase de estabilização e os três blocos da fase de adaptação. Para as medidas de erro absoluto e número de acertos de execução foram utilizados os testes não-paramétricos correspondentes, para verificar as diferenças entre os blocos de tentativas, testes de Friedman para cada grupo e para verificar se havia diferenças entre os grupos, testes $U$ de Mann Whitney em cada bloco.

\begin{tabular}{|c|c|c|c|c|}
\hline \multirow{2}{*}{$\begin{array}{c}\text { Medidas } \\
\text { de } \\
\text { desempenho }\end{array}$} & \multicolumn{2}{|c|}{ Estabilização } & \multicolumn{2}{|c|}{ Adaptação } \\
\hline & $\begin{array}{l}\text { Diferenças } \\
\text { intra-grupo }\end{array}$ & $\begin{array}{l}\text { Diferenças } \\
\text { entre grupo }\end{array}$ & $\begin{array}{l}\text { Diferenças } \\
\text { intra-grupo }\end{array}$ & $\begin{array}{c}\text { Diferenças } \\
\text { entre grupo }\end{array}$ \\
\hline \multirow{7}{*}{$\begin{array}{c}\text { Erro } \\
\text { absoluto }\end{array}$} & PCA & $\mathrm{PCB}<\mathrm{PCA}$ & PCA & \multirow{7}{*}{ Sem diferenças } \\
\hline & $\mathrm{E} 1>\mathrm{E} 2, \mathrm{E} 3, \mathrm{E} 4$ & & $\mathrm{E} 4>\mathrm{A} 1, \mathrm{~A} 2, \mathrm{~A} 3$ & \\
\hline & $\mathrm{e}$ & E1 & $\mathrm{e}$ & \\
\hline & $\mathrm{E} 2>\mathrm{E} 3, \mathrm{E} 4$ & $(\mathrm{z}=-4,620, \mathrm{p}=0,00)$ & $\mathrm{A} 1>\mathrm{A} 2$ & \\
\hline & & $\mathrm{e}$ & & \\
\hline & {$\left[\mathrm{x}^{2}(3, \mathrm{n}=30)=\right.$} & E2 & $\mathrm{x}^{2}(3, \mathrm{n}=30)=$ & \\
\hline & $29,520, p=0,00]$ & $(\mathrm{z}=-3,090, \mathrm{p}=0,00)$ & $20,200, p=0,00]$ & \\
\hline \multirow{5}{*}{$\begin{array}{c}\text { Erro } \\
\text { variável }\end{array}$} & $\mathrm{E} 1>\mathrm{E} 4$ & $\mathrm{PCB}<\mathrm{PCA}$ & $\mathrm{E} 4>\mathrm{A} 1, \mathrm{~A} 2, \mathrm{~A} 3$ & \multirow{5}{*}{ Sem diferenças } \\
\hline & & & e & \\
\hline & {$[F(3 ; 174)=2,7844$,} & $\mathrm{F}(3 ; 174)=2,7844$, & $\mathrm{A} 1>\mathrm{A} 2, \mathrm{~A} 3$ & \\
\hline & $\mathrm{p}=0,04]$ & $\mathrm{p}=0,04]$ & & \\
\hline & & & $\begin{array}{l}\mathrm{F}(3 ; 174)=23,2677 \\
\mathrm{p}=0,00]\end{array}$ & \\
\hline \multirow{10}{*}{$\begin{array}{c}\text { Acertos de } \\
\text { execução }\end{array}$} & PCA & \multirow{10}{*}{ Sem diferenças } & PCA & \multirow{4}{*}{$\begin{array}{l}\text { PCB > PCA } \\
\text { A2 } \\
(z=-2,315, p=0,02)\end{array}$} \\
\hline & $\mathrm{E} 1<\mathrm{E} 2, \mathrm{E} 3$ & & $\mathrm{E} 4<\mathrm{A} 2, \mathrm{~A} 3$ & \\
\hline & {$\left[\mathrm{x}^{2}(3, \mathrm{n}=30)=\right.$} & & $\mathrm{x}^{2}(3, \mathrm{n}=30)=$ & \\
\hline & $12,977, \mathrm{p}=0,00]$ & & $18,544, p=0,00]$ & \\
\hline & & & & \\
\hline & & & PCB & \\
\hline & & & $\mathrm{E} 4<\mathrm{A} 2, \mathrm{~A} 3$ & \\
\hline & & & $\mathrm{A} 2$ \# A1, A3 & \\
\hline & & & $\mathrm{x}^{2}(3, \mathrm{n}=30)=$ & \\
\hline & & & $22,146, \mathrm{p}=0,00]$ & \\
\hline
\end{tabular}



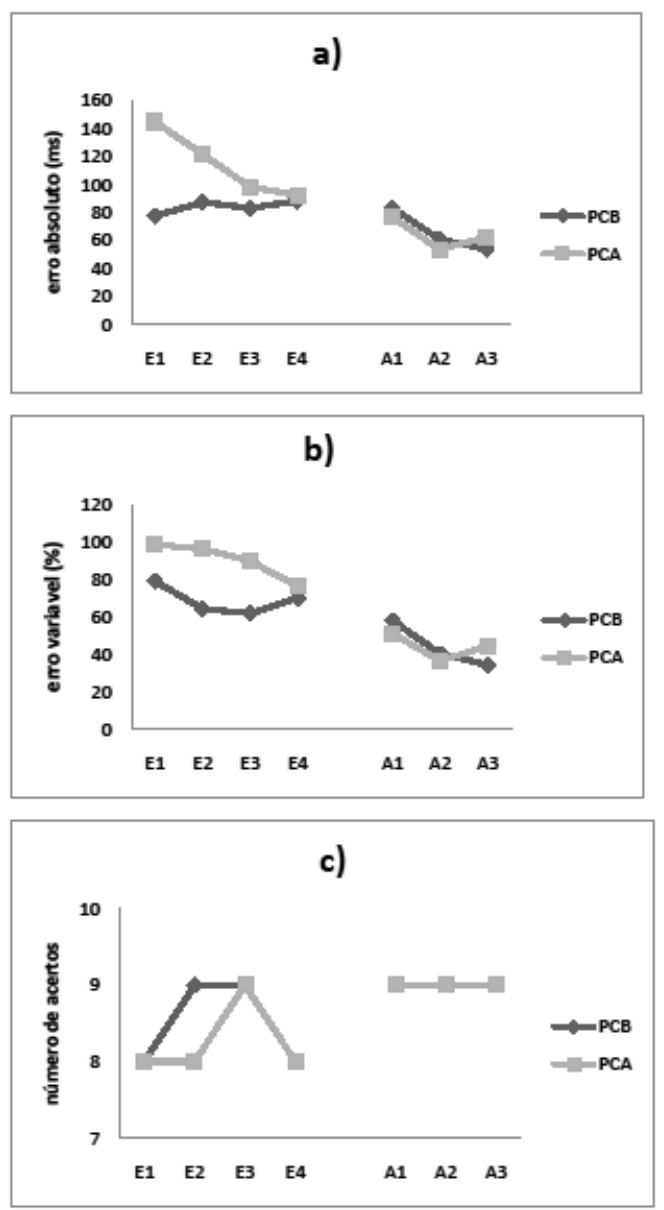

FIGURA 2 -Medianas das medidas de erro absoluto (a), erro variável (b) e número de acertos de execução (c) dos grupos PCB e PCA nos últimos quatro blocos da fase de estabilização (E1 a E4) e nos três blocos da fase de adaptação (A1 a A3).

\section{Medidas de macroestrutura e de microestrutura}

As diferenças estatísticas que embasam as análises a seguir são apresentadas na TABELA 2. Com relação ao TR, o PCB alterou a proporção de tempo de quatro dos cinco componentes (componente 2, 3, 4 e 5 ) ao passo que o PCA alterou apenas do componente 3 durante a fase de estabilização. Apesar disso, a consistência da estrutura formada verificada através da medida de VTR mostrou que o PCB foi mais consistente que o PCA durante a estabilização.

$\mathrm{Na}$ fase de adaptação, os grupos adotaram as mesmas estratégias quanto ao "timing" relativo dos cinco componentes da tarefa. Apenas quando foi verificada a manutenção ou não da estrutura formada durante a fase anterior foram encontradas diferenças de comportamento. O PCB alterou o TR dos componentes 2, 4 e 5 da tarefa, realizando os componentes 2 e 4 mais rapidamente e o componente 5 mais lentamente na fase de adaptação em relação ao último bloco da estabilização. O PCA alterou os componentes $2,3,4 \mathrm{e} 5$, realizando os três primeiros de forma mais rápida e o último de forma mais lenta na adaptação. Portanto, os dois grupos alteraram a estrutura formada anteriormente para se adaptar à nova tarefa (TABELA 3).

A medida de VTR mostrou que os dois grupos comportaram-se de forma semelhante, apresentando melhor consistência nos dois últimos blocos da adaptação do que no final da fase anterior. Os dois grupos diminuíram também a variabilidade ao longo da adaptação, do primeiro para o último bloco. Já a medida de variabilidade do tempo total de movimento (VTTM) mostrou que o PCB aumenta a variabilidade ao longo dos blocos ao passo que o PCA tende a diminuí-la. Assim, o PCA mostrou-se menos variável que o PCB no terceiro e quarto blocos da estabilização. $\mathrm{Na}$ fase de adaptação, os dois grupos apresentaram variabilidade menor do que no final da fase anterior, porém sem diferença entre eles (FIGURA 3). 
TABELA 2 -Diferenças estatísticas significativas intra-grupo e entre grupos dos PCB e PCA das medidas de "timing relativo" dos cinco componentes da macroestrutura (TR1 a TR5), da variabilidade do "timing relativo" da macroestrutura (VTR) e da variabilidade do tempo total de movimento (VTTM) da microestrutura nos últimos quatro blocos da fase de estabilização (E1 a E4) e entre o último bloco da fase de estabilização (E4) e os três blocos da fase de adaptação (A1 a A3).

Na primeira metade da tabela são apresentadas as diferenças estatísticas para cada medida relativas à fase de estabilização, na primeira coluna, as diferenças entre os blocos e, na segunda coluna, as diferenças entre os grupos PCB e PCA. Foram conduzidas análises de variância com medidas repetidas no segundo fator (ANOVAs two way) para todas as medidas nos quatro blocos da fase de estabilização exceto para as medidas de "timing relativo" do primeiro componente (TR1) e para a medida de variabilidade do tempo total de movimento (VTTM), para essas medidas, foram utilizados os testes não-paramétricos correspondentes.

Na segunda metade da tabela são apresentadas as diferenças estatísticas para cada medida relativas à fase de adaptação, na primeira coluna, as diferenças entre os blocos e, na segunda coluna, as diferenças entre os grupos PCB e PCA. Foram conduzidas análises de variância com medidas repetidas no segundo fator (ANOVAs two way) para todas as medidas entre $o$ último bloco da fase de estabilização e os três blocos da fase de adaptação exceto para as medidas de "timing relativo" do segundo componente (TR2) e do quinto componente (TR5) da macroestrutura, em que foram utilizados os testes não-paramétricos correspondentes.

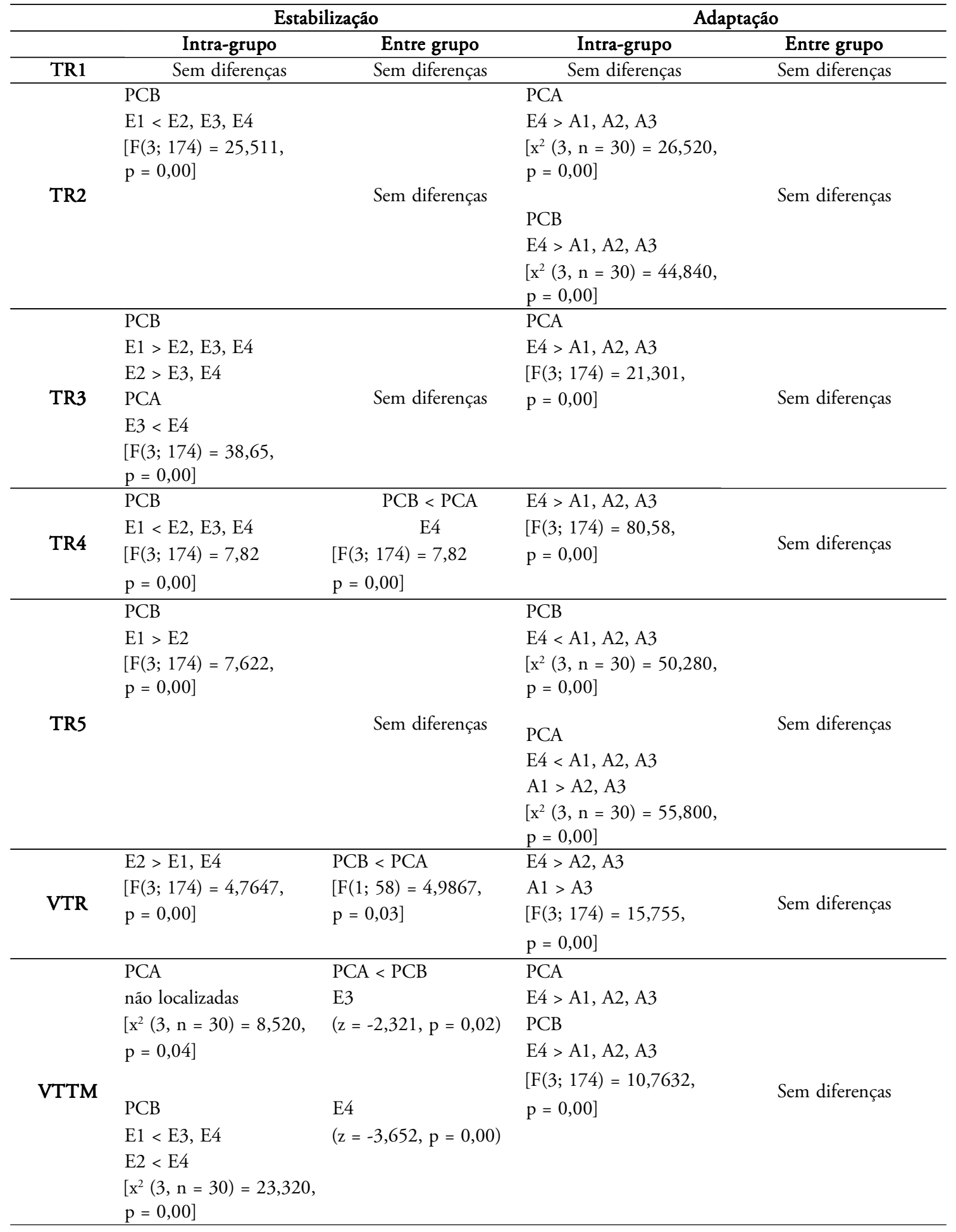


TABELA 3 - Medianas das medidas de "timing relativo" em milisegundos dos cinco componentes (TR1 a TR5) do PCB e do PCA nos últimos quatro blocos da fase de estabilização (E1 a E4) e nos três blocos da fase de adaptação (A1 a A3).

\begin{tabular}{ccccccccc}
\hline & & E1 & E2 & E3 & E4 & A1 & A2 & A3 \\
\hline \multirow{6}{*}{ PCB } & TR1 & 11,87 & 11,32 & 11,46 & 10,79 & 10,66 & 10,75 & 11,00 \\
& TR2 & 19,79 & 21,99 & 22,64 & 22,14 & 20,43 & 20,36 & 20,18 \\
& TR3 & 22,97 & 20,96 & 18,84 & 19,46 & 19,28 & 19,54 & 20,13 \\
& TR4 & 19,85 & 22,07 & 21,93 & 21,89 & 19,31 & 19,69 & 19,38 \\
& TR5 & 24,27 & 23,21 & 23,51 & 24,39 & 28,98 & 29,39 & 28,70 \\
& & & & & & & & \\
& TR1 & 10,57 & 10,10 & 10,30 & 10,67 & 9,75 & 10,89 & 10,61 \\
& TR2 & 22,12 & 21,59 & 21,78 & 21,56 & 19,54 & 19,72 & 20,21 \\
& TR3 & 20,87 & 20,81 & 20,47 & 21,87 & 19,20 & 19,16 & 18,96 \\
& TR4 & 23,28 & 22,71 & 23,01 & 22,36 & 19,45 & 19,27 & 19,45 \\
& TR5 & 21,67 & 22,53 & 23,02 & 22,29 & 31,09 & 30,41 & 29,52 \\
\hline
\end{tabular}
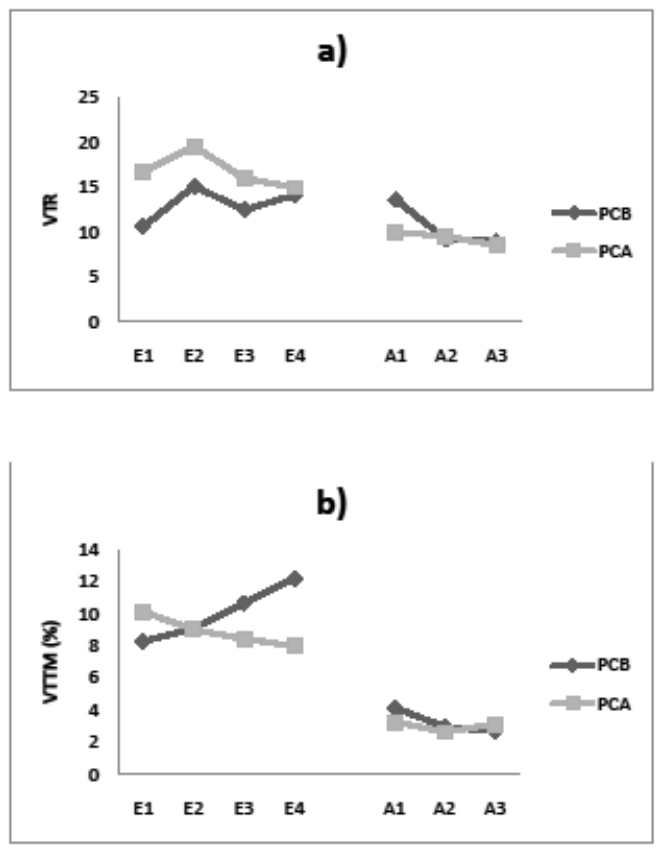

FIGURA 3 - Medianas das medidas de variabilidade do "timing relativo"(VTR) da macroestrutura do PCB e do PCA (a) e das medidas de variabilidade do tempo total de movimento (VTTM) da microestrutura do PCB e do PCA (b) nos últimos quatro blocos da fase de estabilização (E1 a E4) e nos três blocos da fase de adaptação (A1 a A3).

\section{Discussão e conclusões}

Nesse experimento foram investigados os efeitos da combinação da prática constante seguida da prática aleatória e da prática constante seguida da prática por blocos na aquisição de uma habilidade motora quando manipulados os aspectos perceptivo e efetor da tarefa.

A análise dos resultados mostrou que os dois grupos apresentaram o mesmo desempenho na fase de adaptação nas medidas de precisão e de consistência, porém, na medida de número de acertos de execução, o PCB errou significativamente menos que o PCA no segundo bloco da fase de adaptação. $\mathrm{O}$ que mostra que a prática por blocos proporciona melhor adaptação a novas situações que a prática aleatória, contrariando, assim, as predições da interferência contextual. Portanto, esse foi mais um estudo entre tantos outros que não confirma o efeito da interferência contextual 
na transferência para uma nova tarefa quando os aprendizes são inexperientes (BARROS, 2006; Carnahan \& Lee, 1989; CorrêA, 2001; CorrêA et al., 2003; Del Rey, Whitehurst \& Wood, 1983; Del Rey, Wughalter, Du Bois, \& Carnes, 1982; Del Rey, Wughalter \& Whitehurst, 1982; Freudenheim \& Tani, 1995; Heitman \& Gilley, 1989; Lage, Alves, Oliveria, Palhares, Ugrinowitsch \& Benda, 2007; Lage, Vieira, Palhares, Ugrinowitsch \& Benda, 2006; Lee, 1985; Meira Júnior \& Tani, 2001; Pollock \& LeE, 1997; Turnbull \& Dickinson, 1986; UgrinowitsCh, 1997; Ugrinowitsch \& MANoel, 1999; Walter, 2007). Esse efeito foi negado mesmo oferecendo prática constante no início da aprendizagem, condição essa que supostamente favoreceria a formação do programa, como sugerem SHEA, KoHL e Indermill (1990) ou a familiarização com o movimento, como sugerem MAGILl e HALL (1990) e assim potencializaria os efeitos da interferência contextual.

Como já discutido anteriormente a abordagem de Processo Adaptativo considera duas fases na aquisição de habilidades motoras: a estabilização e a adaptação (TANI, 1995, 2005). A primeira representa um processo de busca por uma padronização espaço-temporal dos movimentos e a segunda, a adaptação a novas situações ou a novas tarefas motoras mediante a aplicação das habilidades motoras já adquiridas. Para desencadear ciclos sucessivos de estabilidade são necessários períodos de instabilidade, em que a perturbação exerce função fundamental no processo de mudanças. Segundo o modelo, quando a perturbação é pequena, o sistema é capaz de se adaptar por meio de sua flexibilidade inerente, fazendo uso da redundância do sistema (TANI, 1995, 2005) porém quando a perturbação é grande as adaptações podem ocorrer por meio de modificações na estrutura da habilidade estabelecida, que resultarão numa mudança qualitativa no sistema em um nível superior de complexidade quando bem sucedida. Entretanto, para possibilitar adaptaçōes às demandas ambientais é necessário que a estrutura formada também tenha características flexíveis (MANOEL \& CONNOLLY, 1995; ManOel et al., 2002; TANI, 1995, 2005).

Nesse estudo buscou-se inserir uma grande perturbação ao alterar os aspectos perceptivos e efetores da tarefa na fase de adaptação. Com relação às medidas do programa de ação, os dois grupos formaram uma nova macroestrutura da habilidade para se adaptar a nova tarefa, adaptação esperada, pois essa é considerada como uma grande perturbação. Ao formar a nova estrutura, os grupos também apresentaram valores absolutos similares em relação ao grau de consistência e um aumento dessa consistência ao longo da adaptação. Esse aumento da consistência da nova estrutura formada indica que a aprendizagem é um processo contínuo com sucessivos ciclos de instabilidade e estabilidade assim como preconiza o modelo do Processo Adaptativo.

$\mathrm{O}$ mesmo ocorreu em relação à microestrutura, os dois grupos foram semelhantes, ambos mostrando menor variabilidade na adaptação do que no final da fase anterior, sem apresentar, no entanto, melhora de consistência ao longo da fase de adaptação, ou seja, ainda mantendo um nível de variabilidade na microestrutura, o que poderia ser considerada como uma variabilidade funcional (Manoel \& Connolly, 1995; Manoel et al., 2002; TANI, 1995, 2005).

No início da prática tanto a macro como a microestrutura é inconsistente, com os aspectos espaciais e temporais mal-definidos inclusive na macroestrutura. Consequentemente, o desempenho é inconsistente e descoordenado e raramente a meta do movimento é alcançada. Ao longo da prática, porém, a macroestrutura vai se estabelecendo uma vez que a interação dos componentes se torna padronizada. Depois da sua formação, a macroestrutura restringe a microestrutura, o que deve resultar na diminuição da variabilidade nesse último nível. No entanto, um nível ótimo de graus de variabilidade deve permanecer na microestrutura, pois, caso contrário, o sistema se tornaria rígido demais para responder de forma flexível às demandas internas e do ambiente (ManOel \& CONNOLLY, 1995; MANOEL et al., 2002; TANI, 1995, 2005).

A elaboração de um programa de ação mais complexo envolve a integração de elementos em um novo padrão de ação (TANI, 1995). Esse tipo de mudança só ocorre diante da disponibilidade de elementos no sistema, pois as estruturas mais complexas são desenvolvidas a partir de outras mais simples. Freudenheim e Manoel (1999) afirmam que um maior número de variações disponíveis faz com que o aprendiz mude o objetivo a ser alcançado, colocando-o frente ao desafio de buscar um novo padrão de interação entre os componentes. Essa busca inicia um novo processo de aprendizagem, que vai resultar na aquisição de um programa de ação mais complexo que o anterior.

Em resumo, os dois grupos conseguiram se adaptar a nova tarefa formando um novo programa de ação e o fizeram reorganizando-o de forma semelhante. Porém o PCA levou mais tempo para se adaptar, pois apresentou mais erros de execução do que o PCB. 
Um ponto importante foi o fato dos dois grupos terem sido capazes de se adaptar a perturbação perceptivo-motora. Diferentemente do estudo de UGRINOWITSCH (2003), nesse estudo, os grupos foram preparados para o tipo de perturbação que iriam enfrentar ao realizar a prática variada depois do alcance do critério de desempenho. Assim, com algumas tentativas de prática variada, após a estabilização, foi possível a adaptação aos três tipos de perturbação imposta. Ao oferecer apenas prática constante (UGRINOWITSCH, 2003) foi necessário garantir a superestabilização do desempenho para a adaptação à perturbação perceptivo-motora. Esse resultado mostra que ao aprender habilidades motoras que requerem constantes adaptações perceptivo-motoras é preciso oferecer uma prática que prepare o sistema para essas demandas. Achado tem implicações práticas importantes e merece ser testado em contextos com validade ecológica crescente para ampliar seu poder de generalização.

Um fato interessante foi que o grupo de prática constante-por blocos fez mais alterações na macroestrutura do que o grupo de prática constantealeatória durante a fase de estabilização. Ao contrário do que se poderia imaginar, foi a prática mais repetitiva que proporcionou a exploração de novas possibilidades de execução do movimento. Portanto, uma maior previsibilidade do ambiente pode ter encorajado os aprendizes a fazerem exploraçōes na forma de realizar a tarefa, permitindo também a escolha do momento em que fazê-las. Já a prática aleatória fez com que os aprendizes mantivessem o padrão anterior para que tivessem condiçôes de lidar com a grande instabilidade do ambiente. Esse dado, associado ao fato do PCB ter apresentado melhor desempenho, mostra que menos instabilidade ambiental favorece a aprendizagem motora pelo menos em suas fases iniciais.

A abordagem do Processo Adaptativo focaliza a importância das experiências diversificadas, no sentido de que, quanto maior for o número de elementos disponíveis, maiores são as possibilidades de combinação entre eles para a formação de novas estruturas. Ao contrário da proposta da Interferência Contextual que focaliza exatamente em como os elementos são adquiridos e memorizados. Portanto, pensando apenas em número de elementos no sistema, as duas práticas oferecem quantidades iguais, sendo assim, poder-se-ia esperar uma igualdade entre as práticas. Entretanto, o PCB apresentou melhores resultados que o PCA quanto a desempenho, o que nos leva a pensar no outro aspecto importante para a formação do programa de ação, qual seja, a interação entre os elementos. Assim, a prática variada por blocos oferece melhores condições para o fortalecimento dessas interações, já que exige menos modificaçōes ao longo da prática.

Estudos anteriores realizados sobre estrutura de prática no processo adaptativo (CORRÊA, 2001; CorrêA et al., 2003, 2006, 2007; CorrêA, BENDA $\&$ TANI, 2001) não abordaram a prática em blocos combinada a prática constante como nesse estudo, mas indicaram que a prática que combina menos variação no início da aprendizagem e mais variação, em um segundo momento, favoreceria mais a aprendizagem do que as práticas isoladas com bastante variação (aleatória) ou com pouca variação (prática constante).

Daí o interesse desse estudo em testar qual a combinação que mais favoreceria o processo adaptativo, uma combinação com um grande aumento na instabilidade em um segundo momento (prática constante-aleatória) ou uma combinação com um aumento gradual da instabilidade (prática constante- por blocos).

Uma possível implicação desses resultados para a área profissional seria que aumento da instabilidade ambiental de forma crescente e gradual, primeiro oferecendo prática constante depois prática por blocos nesses momentos iniciais, favoreceria a aprendizagem. Talvez aumentos crescentes na instabilidade em fases posteriores da aprendizagem, como a inserção da prática aleatória, por exemplo, tornariam o sistema mais adaptável e capaz de lidar com perturbações maiores. Entretanto, é apenas uma hipótese que poderia ser testada em futuros estudos.

Em suma, pode-se concluir que, das combinações investigadas, a prática constante seguida da prática variada por blocos foi a que proporcionou melhores resultados na adaptação a uma nova tarefa, quando a perturbação foi apresentada sem variações. Outras questōes que surgem são: condiçōes diferenciadas na fase de adaptação poderiam modificar esses comportamentos? A prática por blocos proporcionou melhores resultados em virtude de sua maior semelhança com as condições em que a tarefa da adaptação foi apresentada? Essa consideração retoma a questão da especificidade da prática que merece ser revista, assim como foi revista, nesse trabalho, a questão da interferência contextual sob um modelo teórico de nãoequilíbrio, trazendo novos e importantes achados. 


\begin{abstract}
The effects of the combination of constant and variable practice in the acquisition of a motor skill

The effects of different practice schedules in the adaptive process of motor skill acquisition were investigated in an experiment involving different regimes of the variable practice. The experimental design consisted of two combined practice groups (constant-block and constant-random) and two learning phases (stabilization and adaptation). Sixty undergraduate students performed a task that consisted of touching response keys sequentially in integration with a visual stimulus. A coincident timing apparatus combined with a serial response device was used. The overall performance was evaluated using three measures: absolute error, variable error and number of correct trials. The relative timing and its variability were used to assess the macrostructure of the action program of the skill while its microstructure was evaluated by absolute movement time and its variability. The variable practice was manipulated both in terms of the visual stimulus velocities and the response patterns. The results showed a better performance for the constant-block practice group in the number of sequence errors in the adaptation phase. Overall results showed a superiority of constantblock practice group relative to the adaptation to the new task.
\end{abstract}

UnITERMS: Practice schedule; Motor skill acquisition; Adaptative process.

\title{
Referências
}

BARROCAL, R.M.; PEREZ, C.R.; MEIRA JUNIOR, C.M.; GOMES, F.R.F.; TANI, G. Faixa de amplitude de conhecimento de resultados e processo adaptativo na aquisição de controle da força manual. Revista Brasileira de Educação Física e Esporte, São Paulo, v.20, p. 111-9, 2006.

BARROS, J.A.C. Estrutura de prática e processo adaptativo em aprendizagem motora: efeitos da especificidade da tarefa. 2006. Dissertação (Mestrado) - Escola de Educação Física e Esporte da Universidade de São Paulo, São Paulo, 2006.

BASTOS, F.H.; ARAÚJO, U.O.; WALTER, C.; FREUDENHEIM, A.M. Liberdade de escolha do aprendiz no processo adaptativo em aprendizagem motora. Revista Brasileira de Educação Física e Esporte, São Paulo, v.21, n.3, p.167-76, 2007. BENDA, R.N. Variabilidade e processo adaptativo na aquisição de habilidades motoras. 2001. Tese (Doutorado) Escola de Educação Física e Esporte, Universidade de São Paulo, São Paulo, 2001.

BENDA, R.N.; CORRÊA, U.C.; OLIVEIRA, D.L.; TANI, G. Variabilidade e processo adaptativo na aprendizagem de uma tarefa de controle da força manual. In: BARREIROS, J.; MELO, F.; SARDINHA, E.B. (Eds.). Percepção \& acção. Lisboa: Ediçôes FMH, 2000. v.3, p.166-182.

BENDA, R.N.; CORREAA, U.C.; OLIVEIRA, D.L.; UGRINOWITSCH, H.; TANI, G. Variabilidade e processo adaptativo na aprendizagem de uma tarefa de posionamento linear com reversão. Revista Brasileira de Ciência e Movimento, São Paulo, v.13, p.7-15, 2005.

CARNAHAN, H.; LEE, T.D. Training for transfer of a movement timing skill. Journal of Motor Behavior, Washington, v.21, p.48-59, 1989.

CORREAA, U.C. Estrutura de prática e processo adaptativo na aquisição de habilidades motoras. 2001. Tese (Doutorado) - Escola de Educação Física e Esporte, Universidade de São Paulo, São Paulo, 2001.

CORRÊA, U.C.; BARROS, J.A.C; MASSIGLI, M.; GONÇALVES, L.A. Prática constante-aleatória e o processo adaptativo de aprendizagem motora: efeitos da quantidade de prática constante. Revista Brasileira de Educação Física e Esporte, São Paulo, v. 21, n.4, p.301-14, 2007.

CORREAA, U.C.; BENDA, R.N.; MEIRA JÚNIOR, C.M.; TANI, G. Practice schedule and adaptive process in the acquisition of a manual force control task. Journal of Human Movement Studies, London, v.44, p.121-38, 2003.

CORREAA, U.C.; BENDA, R.N.; TANI, G. Estrutura de prática e processo adaptativo na aprendizagem do arremesso de dardo de salão. Revista Brasileira de Ciências do Esporte, São Paulo, v.22, p.69-84, 2001.

CORRÊA, U.C.; GONÇALVES, L.A.; BARROS, J.A.C.; MASSIGLI, M. Prática constante-aleatória e aprendizagem motora: efeitos da quantidade de prática constante e da manipulação de exigências motoras da tarefa. Brazilian Journal of Motor Behavior, Rio Claro, v.1, p.41-52, 2006. 
DEL REY, P.; WHITEHURST, M.; WOOD, J.M. Effects of experience and contextual interference on learning and transfer by boys and girls. Perceptual and Motor Skills, Missoula, v.56, p.581-2, 1983.

DEL REY, P.; WUGHALTER, E.; DU BOIS, D.; CARNES, M.M. Effects of contextual interference and retention intervals on transfer. Perceptual and Motor Skills, Missoula, v.54, p.467-76, 1982.

DEL REY, P.; WUGHALTER, E.H.; WHITEHURST, M. The effects of contextual interference on females with varied experience in open sport skills. Research Quarterly for Exercise and Sports, Washington, v.53, n.2, p.108-15, 1982.

FREUDENHEIM, A.M.; MANOEL, E.J. Organização hierárquica e a estabilização de um programa de ação: um estudo exploratório. Revista Paulista de Educação Física, São Paulo, v.13, p.117-96, 1999.

FREUDENHEIM, A.M.; OLIVEIRA, J.A.; CORREAA, U.C.; OLIVEIRA, P.H.; DANTAS, L.E.P.B.T.; MOREIRA, C.R.P.; TANI, G. Efeito da velocidade do estímulo no desempenho de crianças e adolescentes em uma tarefa complexa de timing coincidente. Revista Portuguesa de Ciências do Desporto, Lisboa, v. 5, n. 5, p.160-166, 2005.

FREUDENHEIM, A.M.; TANI, G. Efeitos da estrutura de prática variada na aprendizagem de uma tarefa de "timing" coincidente em crianças. Revista Paulista de Educação Física, São Paulo, v.9, p.87-98, 1995.

HEITMAN, R.J.; GILLEY, W.F. Effects of blocked versus random practice by mentally retarded subjects on learning a novel skill. Perceptual and Motor Skills, Missoula, v.69, p.443-47, 1989.

LAGE, G.M.; ALVES, M.A.F.; OLIVERIA, F.S.; PALHARES, LR.; UGRINOWITSCH, H.; BENDA, R.N. The combination of practice schedules: effects on relative and absolute dimensions of the task. Journal of Human Movement Studies, London, v.52, p.21-35, 2007.

LAGE, G.M.; VIEIRA, M.; PALHARES, L.R.; UGRINOWITSCH, H.; BENDA, R.N. Practice schedules and number of skills as contextual interference factors in the learning of positioning timing tasks. Journal of Human Movement Studies, London, v.50, p.185-200, 2006.

LAI, Q.; SHEA, C.H. Generalized motor program (GMP) learning: effects of reduced frequency of knowledge of results and practice variability. Journal of Motor Behavior, Washington, v.30, p.51-9, 1998.

LAI, Q.; SHEA, C.H.; BRUECHERT, L.; LITTLE, M. Auditory model enhances relative-timing learning. Journal of Motor Behavior, Washington, v.34, p.299-307, 2002.

LAI, Q.; SHEA, C.H.; WULF, G.; WRIGHT, D.L. Optimizing generalized motor program and parameter learning. Research Quarterly for Exercise and Sport, Washington, v.71, p.10-24, 2000.

LEE, T.D. Effects of presentation schedule on retention and prototype formation for kinesthetically presented figures. Perceptual and Motor Skills, Missoula, v.60, p.639-43, 1985.

MAGILL, R.A.; HALL, K.G. A review of the contextual interference effect in motor skill acquisition. Human Movement Science, Amsterdan, v.9, p.241-89, 1990.

MANOEL, E.J. Controles ótimo e adaptativo na aquisição de habilidades motoras seriadas. Revista Paulista de Educação Física, São Paulo, v.6, p.3-20, 1992.

MANOEL, E.J.; BASSO, L.; CORREA, U.C.; TANI, G. Modularity and hierarchical organisation of actions programs in the acquisition of graphic skills. Neuroscience Letters, Amsterdam, v.328, n.3, p.125-36, 2002.

MANOEL, E.J ; CONNOLLY, K.J. Variability and the development of skilled actions. International Journal of Psychophysiology, Amsterdam, v.19, p.129-47, 1995

MEIRA JÚNIOR, C.M. O efeito da interferência contextual na aquisição da habilidade "saque" do voleibol em crianças: temporário, duradouro ou inexistente? 1999. Dissertação (Mestrado) - Escola de Educação Física e Esporte, Universidade de São Paulo, São Paulo, 1999.

MEIRA JÚNIOR, C.M.; TANI, G. The contextual interference effect in acquisition of dart-throwing skill tested on a transfer test with extended trials. Perceptual and Motor Skills, Missoula, v.92, p.910-18, 2001.

PAYNE, V.G. Effects of angle of stimulus approach on coincidence anticipation timing performance. Journal of Human Movement Studies, London, v.13, p.383-90, 1987.

PINHEIRO, J.; CORRÊA, U.C. Desempenho em uma tarefa complexa de timing coincidente com desaceleração do estímulo visual em indivíduos de diferentes idades. Revista Brasileira de Educação Física e Esporte, São Paulo, v.19, n.1, p.61-70, 2005.

POLLOCK, B.J.; LEE, T.D. Dissociated contextual interference effects in children and adults. Perceptual and Motor Skills, Missoula, v.84, p.851-858, 1997.

SHEA, C.H., KOHL, R.; INDERMILL, C. Contextual interference: contributions of practice. Acta Psychologica, Amsterdam, v.73, p.145-157, 1990.

SHEA, C.H.; LAI, Q.; WRIGHT, D. L.; IMMINK, M.; BLACK, C. Consistent and variable practice conditions: effects on relative and absolute timing. Journal of Motor Behavior, Washington, v.33, p.139-52, 2001. 
TANI, G. Hierarchical organization of action programme in the acquisition of a grafic skill. Sheffield: [s.ed.], 1995. (Relatório final de atividades de Pós-doutorado, University of Sheffield).

TANI, G. (Org.). Comportamento motor: aprendizagem e desenvolvimento. Rio de Janeiro: Guanabara Koogan, 2005. TANI, G.; MEIRA JUNIOR, C.M.; GOMES, F. R. F. Frequência, precisão e localização temporal de conhecimento de resultados e o processo adaptativo na aquisição de uma habilidade motora de controle de força manual. Revista Portuguesa de Ciências do Desporto, Lisboa, v.5, p.59-68, 2005.

TURNBULL, S.D.; DICKINSON, J. Maximizing variability of practice: a test of schema theory and contextual interference theory. Journal of Human Movement Studies, London, v.12, p.201-213, 1986.

UGRINOWITSCH, H. Interferência contextual: manipulação de programas e parâmetros na aquisição da habilidade "saque" do voleibol. 1997. Dissertação (Mestrado)- Escola de Educação Física e Esporte, Universidade de São Paulo, São Paulo, 1997.

. Efeito do nível de estabilização do desempenho e do tipo de perturbação no processo adaptativo na aprendizagem motora. 2003. Tese (Doutorado)- Escola de Educação Física e Esporte, Universidade de São Paulo, São Paulo, 2003. UGRINOWITSCH, H.; COCA, A.A.; TERTULIANO, I.W.; GIMENEZ, R.; PEREIRA, F.A.S. Freqüência de feedback como um fator de incerteza no processo adaptativo em aprendizagem motora. Revista Brasileira de Ciência e Movimento, São Paulo, v.11, n.2, p.41-7, 2003.

UGRINOWITSCH, H.; CORREAA, U.C.; TANI, G. Perturbação perceptiva e processo adaptativo na aprendizagem de uma tarefa de timing coincidente. Revista Brasileira de Educação Física e Esporte, São Paulo, v.19, n.4, p.24-30, 2005. UGRINOWITSCH, H.; MANOEL, E.J. Interferência contextual: variação de programa e parâmetro na aquisição da habilidade motora saque do voleibol. Revista Paulista de Educação Física, São Paulo, v.13, n.2, p.197-216, 1999.

VAN ROSSUM, J.H.A. Schmidt's schema theory: the empirical base of variability of practice hypothesis- a critical analysis. Human Movement Science, Amsterdam, v.9, p.387-435, 1990.

WALTER, C. Estrutura de prática e liberdade de escolha na aprendizagem de habilidades motoras. 2007. Dissertação (mestrado)- Escola de Educação Física e Esporte, Universidade de São Paulo, São Paulo, 2007.

WULF, G. The learning of generalized motor programs and motor schemata: effects of KR relative frequency and contextual interference. Journal of Human Movement Studies, London, v.23, p.53-76, 1992.

WULF, G.; LEE, T.D. Contextual interference in movement of the same class: differential effects on program and parameter learning. Journal of Motor Behavior, Washington, v.25, p.254-63, 1993.

\section{Agradecimentos}

Ao CNPq pela bolsa de Produtividade em Pesquisa concedida ao segundo autor.

\begin{tabular}{r|l} 
ENDEREÇO & \\
Rejane Paroli & Recebido para publicação: 13/02/2008 \\
1a. Revisão: 14/10/2008 \\
R. Praia do Castelo, 65 - apto. 34A & 2a. Revisão: 09/06/2009 \\
04362-020 - São Paulo - SP - BRASIL & Aceito: 20/07/2009 \\
e-mail: paroli@usp.br & \\
\hline
\end{tabular}

